\title{
Classification of Chest X-Ray Images using Wavelet and MFCC Features and Support Vector Machine Classifier
}

\author{
Hamza Abu Owida \\ Medical Engineering Department \\ Faculty of Engineering \\ Al-Ahliyya Amman University \\ Amman, Jordan \\ h.abuowida@ammanu.edu.jo
}

\author{
Amani Al-Ghraibah \\ Medical Engineering Department \\ Faculty of Engineering \\ Al-Ahliyya Amman University \\ Amman, Jordan \\ a.ghraibah@ammanu.edu.jo
}

\author{
Muneera Altayeb \\ Electronics and Communications Engineering Department \\ Faculty of Engineering \\ Al-Ahliyya Amman University \\ Amman, Jordan \\ m.altayeb@ammanu.edu.jo
}

\begin{abstract}
The shortage and availability limitation of RT-PCR test kits and is a major concern regarding the COVID-19 pandemic. The authorities' intention is to establish steps to control the propagation of the pandemic. However, COVID-19 is radiologically diagnosable using $x$-ray lung images. Deep learning methods have achieved cutting-edge performance in medical diagnosis software assistance. In this work, a new diagnostic method for detecting COVID-19 disease is implemented using advanced deep learning. Effective features were extracted using wavelet analysis and Mel Frequency Cepstral Coefficients (MFCC) method, and they used in the classification process using the Support Vector Machine (SVM) classifier. A total of 2400 Xray images, 1200 of them classified as Normal (healthy) and 1200 as COVID-19, have been derived from a combination of public data sets to verify the validity of the proposed model. The experimental results obtained an overall accuracy of $98.8 \%$ by using five wavelet features, where the classification using MFCC features, MFCC-delta, and MFCC-delta-delta features reached accuracy around $97 \%$ on average. The results show that the proposed model has reached the required level of success to be applicable in COVID 19 diagnosis.
\end{abstract}

Keywords-chest X-ray images; classification of chest diseases; covid-19; feature extraction; Support Vector Machine (SVM)

\section{INTRODUCTION}

Rarely the danger of disease involved such an extensive amount of our thought as the COVID-19 pandemic. The disease's growth, transmission, and increased mortality make the improvement of prevention important. COVID-19 virus came to light in December 2019 in Wuhan Province, China, and the ensuing pandemic influenced most countries with extensive worldwide health, economic, and personal effects [1].
In order to control the disease, identification and early and follow-up quarantine, are important. The early COVID-19 diagnosis is significantly crucial. The key tool for the diagnosis of COVID-19 disease is actually a reverse transcriptasepolymerase chain reaction (RT-PCR) [2]. Currently, the RTPCR test is considered a time consuming and strenuous with complicated manual procedure. Moreover, there is a shortage and availability limitation of RT-PCR test kits [3]. Therefore, an integrated solution system, which is both rapid and reliable, is urgently needed for COVID-19 diagnosis. Radiological imaging is an effective and important tool for the detection of COVID-19 in addition to the RT-PCR method [4]. Medical imaging method is complicated because the radiologist must advertently identify the white spots that contain water and pus, which is time consuming and troublesome. Also, a radiologist or doctor may often misdiagnose other conditions including pulmonary tuberculosis as COVID-19. For that reason, recently developed artificial intelligence-driven automatic diagnostic systems based on machine and deep learning result in quicker and more reliable COVID-19 detection and can be considered as alternatives to manual testing [2-4].

\section{THE STATE OF THE ART AUTOMATED METHODS IN COVID-19 DETECTION}

There are several studies in the published literature which are based on machine learning applications to detect and diagnose COVID-19. These state of the art approaches are based on machine and deep learning approaches by using chest X-ray images. Machine and deep learning systems are frequently used to automatically extract specific classification features for the purpose object. Authors in [4] established a Convolutional Neural Network (CNN)-based model for 
COVID-19 diagnosis. They used 3 different groups of X-ray images, i.e. COVID 19, pneumonia, and normal/healthy. The researchers managed to achieve a classification accuracy of $92.6 \%$ with images from all groups, while authors in [5] reported $87 \%$ accuracy in a similar study. Authors in [6] evaluated the performance of different CNNs (VGG19, Inception, MobileNetV2, Xception, and InceptionResNetV2) in COVID-19 detection. They used three different groups of Xray images comprising of COVID 19, pneumonia, and normal and the highest achieved accuracy was $96.78 \%$. Authors in [7] built up a Bayesian Convolutional Neural Network (BCNN) architecture for COVID-19 detection with an accuracy score of 92.9\%. Authors in [8] developed a CNN model termed as CoroDet for automatic COVID-19 detection by using chest Xray images with accuracy of $94.2 \%$ for COVID, normal, and non-COVID viral pneumonia classes [8]. Authors in [9] proposed a CNN model, and Support Vector Machine (SVM) with the linear kernel function to classify COVID-19 and healthy chest X-ray images and reached $94.7 \%$ accuracy. Authors in [10] developed two cascade networks, SEMEResNet50 and SEME-DenseNet169, to assess three classes of chest X-ray images: normal, bacterial, and COVID-19 pneumonia with $85.6 \%$ accuracy score in classification of pneumonia infection. Authors in [11] proposed a CNN model and transfer learning for COVID-19 detection for 3 classes, normal, bacterial, and COVID-19 pneumonia with $95.69 \%$ precision by using ResNet-50 pretrained model. Authors in [12] developed an integrated deep learning model focused on a capsule network and used a four-class dataset of chest X-ray images, achieving 95.17\% accuracy. Authors in [13] designed a decently pretrained ResNet152 architecture using transfer learning for the identification of COVID-19 and attained a success rate of $87 \%$. Authors in [14] proposed a deep learningbased approach for the diagnosis of COVID-19 using X-ray images for infected patients and achieved $95.38 \%$ accuracy using the ResNet50 architecture with SVM classifier.

The current study aims to implement a new model for COVID-19 disease detection by using advanced deep learning technique. Effective features were extracted using wavelet analysis and Mel Frequency Cepstral Coefficients (MFCC) method, and in the classification process the SVM classifier was utilized. A total of 2400 X-ray images, 1200 Normal (healthy) and 1200 COVID-19, were derived from a combination of public data sets to verify the validity of the proposed model. With regard to the pandemic outbreak, the proposed study seeks to close the gap between the small number of highly qualified radiologists and the increasing need for chest $\mathrm{x}$-ray interpretation.

\section{Methodology}

\section{A. Feature Extraction}

COVID 19 symptoms can be seen in lungs, both in the peripheral and subpleural areas, particularly in lower lobes and posterior segments. In X-ray images, the appearance and consolidation of bilateral nodular and peripheral ground glass lenses should serve as a warning for radiologists of the potential for COVID-19 [15]. However, X-ray image diagnosis and examination of COVID-19 symptom consumes time and relies on the existence of medical experts. Hence, computer assistance is important in helping the medical staff to detect COVID-19 in X-ray images [4]. Many methods for extracting features are used in the literature. This study aims to apply methods for detecting COVID-19 from X-ray images. These methods are described in detail in the next section.

\section{1) Wavelet Features}

In image processing, 2-Dimensional Discrete Wavelet Transformation (2D DWT) is a powerful image analysis, denoising, and image segmentation method. When the original image is transformed by low pass and high pass filters in the $x$ and $y$ directions, the 2D DWT is computed. The obtained images are down-sampled to half of the original image dimensions. With high pass and low pass filters, the resulting images are convoluted again. The four sub-band acquired images comprise the approximation coefficient (containing the maximum image information), horizontal, diagonal and vertical image information, and the approximation coefficient (containing the maximum image information) [16, 17]. Three image details are being employed to determine the energy of any decomposition level by applying the absolute values for the wavelet coefficients (the highpass images). Then the total energy for the three high pass images is computed. Five energy values corresponding to each of 5 decomposition levels are extracted.

\section{2) Mel Frequency Cepstral Coefficient (MFCC) Features}

MFCC is a very popular and efficient technique for signal processing where the frequency bands are distributed depending on the Mel-scale [15, 18].

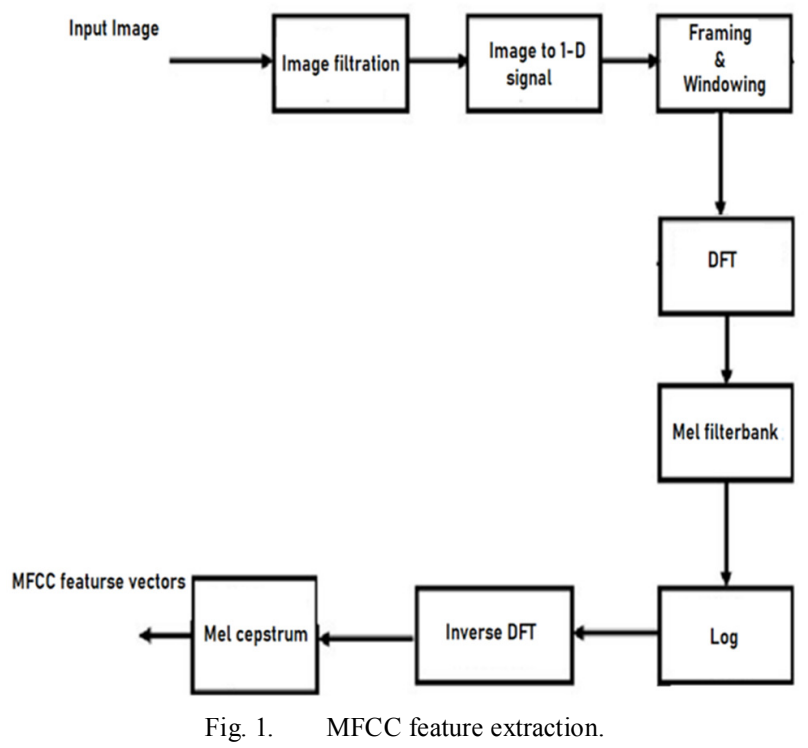

This research presents a new purpose of working with MFCC by using it to extract the features of chest X-ray images. The present technique is based on converting the chest X-ray images into one dimensional (1-D) signal and then extracting MFCC, delta-MFCC, and delta-delta MFCC from the converted 1-D signal. The output after applying MFCC is a matrix. In this output matrix the rows represent the corresponding frame numbers and the columns represent the 
corresponding feature vector coefficients. Classification is performed by using SVM. The steps involved in the MFCC feature extraction are summarized in Figure 1 [19].

\section{3) Framing and Windowing}

The resulting 1-D signal is a non-stationary signal, therefore analysis should always be performed by blocking the signal into possibly overlapping frames, so that the signal is constant [20]. In this step, the continuous 1-D signal is broken into 60 frames of $N=2000$ samples, with consecutive frames separated by $L=512$ samples. The adjacent frames are overlapped by $N-L$ samples, which is around $74.4 \%$. Windowing is done by using a hamming window represented by $w(n)$ multiplied by the input signal represented with $x(n)$. The output signal is represented by (1):

$$
x_{i}(n)=x(n) w\left(n-m_{i}\right), i=0, \ldots, k-1
$$

where $k$ is the number of frames and $n$ is the number of samples by which the window is shifted in order to yield the $i$ th frame then taking the DFT of the resulting signal [21].

\section{4) Mel-Spectrum}

The resulting spectrum of Fourier transformed signal is given as input to a Mel-scale filter bank that consists of 24 filters. The coefficients of each Short Time Fourier Transform (STFT) are multiplied by the corresponding filter gain to know the approximation of the existing energy at each spot with the help of a triangular overlapping window. These energies are also known as the Mel spectrum and can be used for calculating the first 13 coefficients using DCT. A popular formula to convert $f$ in hertz into $f_{m e l}$ is given in (2) $[20,21]$ :

$$
f_{m e l}=2595 \log _{10}\left(1+\frac{f}{700}\right)
$$

\section{5) Discrete Cosine Transform (DCT)}

The DCT applied to the transformed Mel frequency coefficients produced a set of MFCC capestral coefficients which are usually referred to as stationary features.

\section{6) Dynamic MFCC Features}

The cepstral coefficients are usually referred to as static features, since they only contain information from a given frame. The extra information about the temporal dynamics of the signal is obtained by computing the first and second derivatives of the cepstral coefficients. The first-order derivative is called delta coefficients (delta-MFCC), and the second-order derivative is called delta-delta coefficients (delta-delta-MFCC). In this work, 39 features are extracted from each method [22]. Figures 2 and 3 show the MFCCs coefficients for the normal chest and chest infected with Coronavirus. The $x$-axis represents the number of MFCCs extracted from the input signal and the $y$-axis represents the feature values for each frame. In this work, the first 13 MFCC coefficients were used and the rest were discarded. The lower order coefficients contain most of the information about the overall spectral shape according to the feature values shown in Figures 2 and 3. We can observe the difference in MFCCs.

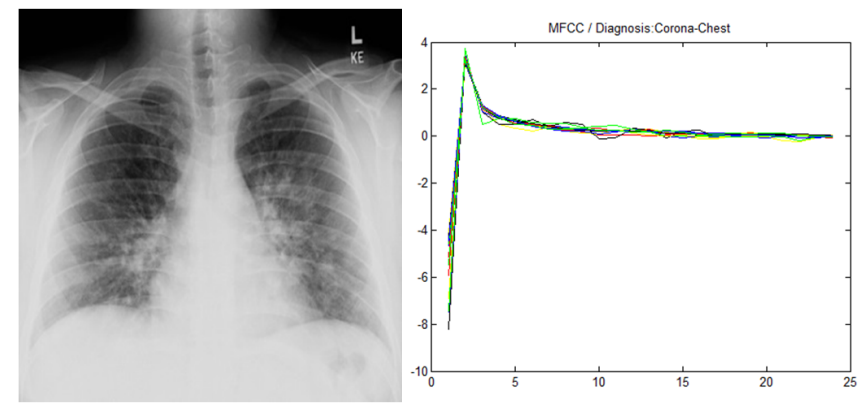

Fig. 2. MFCC features for an infected patient's X-ray chest image.
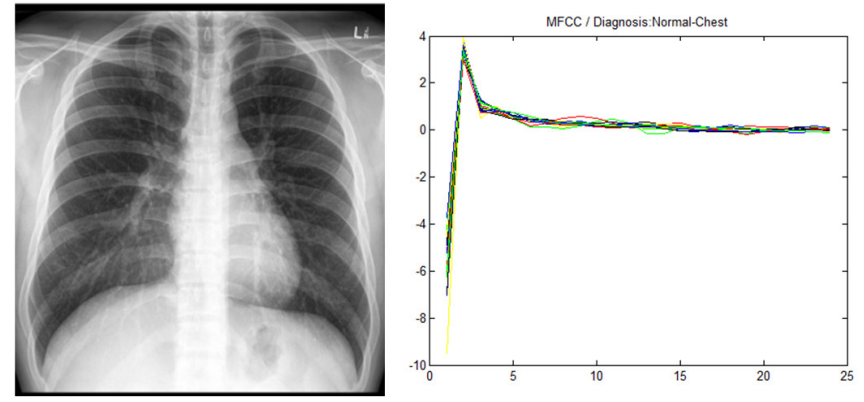

Fig. 3. MFCC features for normal patient's X-ray chest image.

\section{B. Classification}

The purpose of supervised machine learning is to build a model that makes predictions based on evidence in the presence of uncertainty. The machine learns from the results when adaptive algorithms classify data patterns. The computer improves its prediction performance when exposed to more observations. Particularly, a learning algorithm uses a known array of input data and known data responses (classes) and trains a model to provide a reliable prediction of how new data will respond $[22,23]$. In this study, the input X-ray image that has COVID-19 disease was addressed class 1 and the image without a disease was addressed as class 0 . Figure 4 shows a simple description of the SVM.

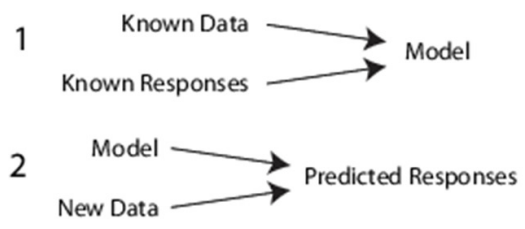

Fig. 4. General description of SVM

The entire set of input data can indeed be termed as a heterogeneous matrix, since the matrix rows are referred to as observations or instances and ach comprises of various measurements for a subject. Matrix columns are defined as predictors or characteristics, which are calculated by each subject [24]. Herein, the observations are X-ray images of human chest and the features of each image are set in columns. The data matrix includes one row of features extracted from each image as shown in (3): 


$$
\left[\begin{array}{ccc}
f 1,1 & \cdots & f 1, n \\
\vdots & \ddots & \vdots \\
f m, 1 & \cdots & f m n
\end{array}\right]
$$

where $n$ is the number of extracted features and $m$ is the number of images.

All supervised learning approaches begin with an input data matrix. The data were arranged in such a way that one observation was made in each row in the feature matrix and one variable or indicator in each column. In this step, each Xray image in the data set has been extracted and organized in a matrix for each image, called a data matrix. Each row has 5 characteristics related to the 5 wavelet features previously mentioned and another 39 features from each MFCC method. Cross-validation is a statistical approach for the computation and comparison of learning algorithms through the division of data into two segments: the first one for model creation and the other for model validation. The essential cross-validation method is a k-fold cross-validation [24, 25]. In this study, a 10fold cross-validation approach was used in which the Matlab software randomly divides the data into 10 sets of the same size, and then trains the SVM classification on the 9 sets. The previous steps are repeated 10 times and the method incorporates generalization statistics from each fold.

\section{Performance Evaluation}

In this study, X-ray images were used for the diagnosis of COVID-19. The total number of chest images used in this work is 2400 , of which 1200 are images with COVID-19 while the rest are images from normal lungs. The terms used in the confusion matrix (Table I) can briefly be described as: True Positive (TP): true event system classified as true, True Negative (TN): false event classified as false, False Positive (FP): false event classified as true, and False Negative (FN): true event classified as false $[25,26]$.

TABLE I. CONFUSION MATRIX

\begin{tabular}{|c|c|c|}
\hline \multirow{2}{*}{$\begin{array}{c}\text { Confusion } \\
\text { matrix }\end{array}$} & \multicolumn{2}{|c|}{ Patient case } \\
\cline { 2 - 3 } Normal & Normal & Abnormal \\
\hline Abnormal & FN & FP \\
\hline
\end{tabular}

Accuracy $(A C)$ refers to the probability that the system classification is correct $[22,23]$ :

$$
A C=\frac{T P+T N}{(T P+F P+T N+F N)} * 100
$$

Sensitivity (True Positive Rate $(T P R)$ ) and Specificity (True Negative Rate $(T N R)$ ) are also calculated from the confusion matrix using (5), and (6) respectively $[25,26]$ :

$$
\begin{gathered}
T P R=\frac{\mathrm{TP}}{\mathrm{TP}+\mathrm{FN}} \\
T N R=\frac{\mathrm{TN}}{\mathrm{TN}+\mathrm{FP}} \\
\text { IV. RESULTS }
\end{gathered}
$$

\section{RESUlts}

In this section, the results of the classification using wavelet features and using MFCC features are presented. As mentioned above, 1200 normal and 1200 coronavirus X-ray images were considered. The data were collected from different publicly accessible datasets, online sources, and published papers. They form the database of COVID-19 X-ray images in [27].

\section{A. Classification using Wavelet Features}

The result confusion matrix of using wavelet features in the classification process is shown in Table II, where the $A C$ is equal to $98.83 \%$, TNR is $99.75 \%$, and $T P R$ is $97.95 \%$.

TABLE II. CONFUSION MATRIX USING WAVELET FEATURES

\begin{tabular}{|l|c|c|}
\hline \multirow{2}{*}{$\begin{array}{c}\text { Confusion } \\
\text { matrix }\end{array}$} & \multicolumn{2}{|c|}{ Patient case } \\
\cline { 2 - 3 } & Normal & Abnormal \\
\hline Normal & 1197 & 3 \\
\hline Abnormal & 25 & 1175 \\
\hline
\end{tabular}

\section{B. Classification using MFCC Features}

This section includes 3 experiments, the first one is classification of the X-ray chest images into normal and corona cases using MFCC features, where the second and the third experiments are accomplished using MFCC-delta and MFCCdelta-delta features.

\section{1) MFCC Features}

The result confusion matrix of using MFCC features in the classification process is shown in Table III, where $A C$ is $95.46 \%, T N R$ is $94.17 \%$, and $T P R$ is $96.82 \%$.

\section{TABLE III. CONFUSION MATRIX USING MFCC FEATURES}

\begin{tabular}{|l|c|c|}
\hline \multirow{2}{*}{$\begin{array}{c}\text { Confusion } \\
\text { matrix }\end{array}$} & \multicolumn{2}{|c|}{ Patient case } \\
Normal & Abnormal \\
\hline Normal & 1128 & 72 \\
\hline Abnormal & 37 & 1163 \\
\hline
\end{tabular}

\section{2) MFCC Delta Features}

The result confusion matrix of using MFCC-delta features in the classification process is shown in Table IV, where $A C$ is equal to $97.75 \%$, TNR is $97.43 \%$, and $T P R$ is $98.07 \%$.

TABLE IV. CONFUSION MATRIX USING MFCC-DELTA FEATURES

\begin{tabular}{|l|c|c|}
\hline \multirow{2}{*}{$\begin{array}{c}\text { Confusion } \\
\text { matrix }\end{array}$} & \multicolumn{2}{|c|}{ Patient case } \\
\cline { 2 - 3 } & Normal & Abnormal \\
\hline Normal & 1169 & 31 \\
\hline Abnormal & 23 & 1177 \\
\hline
\end{tabular}

\section{3) MFCC Delta Delta Features}

The result confusion matrix of using MFCC-delta-delta features in the classification process is shown in Table $\mathrm{V}$, where the $A C$ is $97.83 \%$, TNR is $97.52 \%$, and $T P R$ is $98.15 \%$.

TABLE V. CONFUSION MATRIX USING MFCC-DELTA-DELTA
FEATURES
\begin{tabular}{|l|c|c|}
\hline Confusion & \multicolumn{2}{|c}{ Patient case } \\
\cline { 2 - 3 } matrix & Normal & Abnormal \\
\hline Normal & 1170 & 30 \\
\hline Abnormal & 22 & 1178 \\
\hline
\end{tabular}

\section{Comparison}

From the presented results, classifying the X-ray images using wavelet features gave higher accuracy than using the three types of MFCC features. Also, MFCC-delta-delta 
provided higher accuracy than the other types of MFCC features.

The number of features used in the classification process is not necessary to be high, but the type of the method used to extract these features is most important to get higher accuracy. From the results, we found that using only 5 effective features (wavelet features) gave better results than using 39 MFCC features. The consumed computational time in the feature extraction process and classification experiment was about 10 minutes. Inspired by the previous research studies, the current work used advanced deep learning techniques to introduce a new model for the detection of COVID-19. The extracted effective features using wavelet analysis and MFCC were used with the SVM classification technique. A hybrid of public data sets have been used to validate the value of the proposed model by a total of $2400 \mathrm{X}$-ray images categorized as 1200 normal images and 1200 images of patients infected with COVID-19. The proposed method aims to help close the gap between the smaller number of highly trained radiologists and the growing need for chest X-ray interpretation in relation to the pandemic outbreak.

\section{DISCUSSION}

Early diagnosis of the coronavirus condition is significant in order to minimize the probability of pandemic transmission. Automated early disease diagnosis can be obtained using radiological images by using artificial intelligence-based image processing approaches. Many studies have recently been carried out based on machine learning models for COVID-19 detection. Mostly, in the present state of the increasing prevalence of COVID-19 cases, many researches focus on clinical characteristics. Deep Learning is a combination of machine learning approaches based primarily on automated image detection and classification. The classification and detection of the purposed object are the dual major objectives in which deep learning is implemented. The progress of machine learning has significant benefits for patient assessment and the improvement of computer-aided systems. Table VI summarizes the methods for automatic detection of coronavirus cases where the proposed method for detecting COVID-19 using X-ray images is compared with the methods used in previous studies. The model results demonstrated in the present research have been shown to be superior than the ones of earlier researches. In this work, a new diagnostic method for detecting COVID-19 disease is implemented using advanced deep learning. Effective features were extracted using wavelet analysis and MFCC method, and they were used in the classification process with the SVM classifier. In order to test the proposed model, publicly available X-ray images used in previous COVID-19 studies were utilized. In the experimental testing of the proposed approach, the highest performing effective properties were selected using the current model. Testing results show an $A C$ score of $98.8 \%$ achieved using wavelet features and demonstrated that the current proposed model is more successful than earlier studies carried out for the detection of COVID-19 using X-ray imaging.

The proposed work has several certain limitations. First of all, the network design could be improved to increase the sensitivity or TP rate of COVID-19 detection. In the current workflow, if the stagel model misclassifies a viral pneumonia image as healthy or bacterial pneumonia, the further detection of COVID-19 or other viruses completely misses. Also, the limited number of COVID-19 images makes it more difficult to train the deep learning models from scratch, which is overcome in the current study by using the deep transfer learning method. The present work is carried out with images from Kaggle [27]. The work can be extended with a greater number of images of COVID-19 form other databases.

TABLE VI. RESULT COMPARISON OF THE PROPOSED MODEL AND PREVIOUS STUDIES

\begin{tabular}{|c|c|c|c|}
\hline Study / Year & Methods and models & No of classes & $\boldsymbol{A C}$ \\
\hline$[4] / 2020$ & CNN/COVID-Net & 3 & $92.6 \%$ \\
\hline$[5] / 2020$ & CNN/ DarkCovid-Net & 3 & $87 \%$ \\
\hline$[6] / 2020$ & Deep transfer/VGG 19 & 3 & $96.8 \%$ \\
\hline$[7] / 2020$ & CNN/ResNet50 & 4 & $88.39 \%$ \\
\hline$[8] / 2021$ & CNN/ CoroDet & 3 & $94.2 \%$ \\
\hline$[9] / 2021$ & CNN/SVM & 2 & \\
\hline$[10] / 2021$ & $\begin{array}{c}\text { Cascade networks SEME- } \\
\text { ResNet50 and SEME- } \\
\text { DenseNet169 }\end{array}$ & 3 & $85.6 \%$ \\
\hline$[11] / 2020$ & CNN/ transfer learning & 3 & $95.69 \%$ \\
\hline$[12] / 2020$ & CNN-based Capsule Networks & 4 & $95.17 \%$ \\
\hline$[13] / 2020$ & CNN/ResNet152 & 2 & $87 \%$ \\
\hline$[14] / 2020$ & ResNet50 and SVM & 2 & $95.38 \%$ \\
\hline Proposed & Wavelet and MFCC and SVM & 2 & $97 \%$ \\
\hline
\end{tabular}

\section{CONCLUSION}

The experimental results show that the proposed application of using wavelet for chest X-ray images feature extraction has very good accuracy, around $98.8 \%$, and hence can be used for application in combination with other techniques such as the MFCC to increase the accuracy rate and to make it more efficient. In future research, a mobile Web-based framework is expected to be build that will assist health professionals in their efforts to identify instances of COVID-19 as well as other illnesses. Furthermore, the studies in this field will be investigated by other AI models and attention modules.

\section{REFERENCES}

[1] J. F.-W. Chan et al., "A familial cluster of pneumonia associated with the 2019 novel coronavirus indicating person-to-person transmission: a study of a family cluster," The Lancet, vol. 395, no. 10223, pp. 514-523, Feb. 2020, https://doi.org/10.1016/S0140-6736(20)30154-9.

[2] V. M. Corman et al., "Detection of 2019 novel coronavirus (2019-nCoV) by real-time RT-PCR," Eurosurveillance, vol. 25, no. 3, Jan. 2020, Art. no. 2000045, https://doi.org/10.2807/1560-7917.ES.2020.25.3.2000045.

[3] O. Vandenberg, D. Martiny, O. Rochas, A. van Belkum, and Z. Kozlakidis, "Considerations for diagnostic COVID-19 tests," Nature Reviews Microbiology, vol. 19, no. 3, pp. 171-183, Mar. 2021, https://doi.org/10.1038/s41579-020-00461-z.

[4] L. Wang, Z. Q. Lin, and A. Wong, "COVID-Net: a tailored deep convolutional neural network design for detection of COVID-19 cases from chest X-ray images," Scientific Reports, vol. 10, no. 1, Nov. 2020, Art. no. 19549, https://doi.org/10.1038/s41598-020-76550-z.

[5] T. Ozturk, M. Talo, E. A. Yildirim, U. B. Baloglu, O. Yildirim, and U. Rajendra Acharya, "Automated detection of COVID-19 cases using deep neural networks with X-ray images," Computers in Biology and Medicine, vol. 121, Jun. 2020, Art. no. 103792, https://doi.org/ 10.1016/j.compbiomed.2020.103792.

[6] I. D. Apostolopoulos and T. A. Mpesiana, "Covid-19: automatic detection from $\mathrm{X}$-ray images utilizing transfer learning with 
convolutional neural networks," Physical and Engineering Sciences in Medicine, vol. 43, no. 2, pp. 635-640, Jun. 2020, https://doi.org/ 10.1007/s13246-020-00865-4.

[7] B. Ghoshal, A. Tucker, B. Sanghera, and W. L. Wong, "Estimating uncertainty in deep learning for reporting confidence to clinicians in medical image segmentation and diseases detection," Computational Intelligence, vol. 37, no. 2, pp. 701-734, 2021, https://doi.org/ 10.1111/coin. 12411

[8] D. Lv, W. Qi, Y. Li, L. Sun, and Y. Wang, "A cascade network for Detecting COVID-19 using chest X-rays," arXiv:2005.01468 [cs, eess], May 2020, Accessed: Jun. 03, 2021. [Online]. Available: http://arxiv.org/abs/2005.01468.

[9] I. Katsamenis, E. Protopapadakis, A. Voulodimos, A. Doulamis, and N. Doulamis, "Transfer Learning for COVID-19 Pneumonia Detection and Classification in Chest X-ray Images," in 24th Pan-Hellenic Conference on Informatics, New York, NY, USA, Nov. 2020, pp. 170-174, https://doi.org/10.1145/3437120.3437300.

[10] A. M. Ismael and A. Şengür, "Deep learning approaches for COVID-19 detection based on chest X-ray images," Expert Systems with Applications, vol. 164, Feb. 2021, Art. no. 114054, https://doi.org/ 10.1016/j.eswa.2020.114054

[11] E. Hussain, M. Hasan, M. A. Rahman, I. Lee, T. Tamanna, and M. Z. Parvez, "CoroDet: A deep learning based classification for COVID-19 detection using chest X-ray images," Chaos, Solitons \& Fractals, vol. 142, Jan. 2021, Art. no. 110495, https://doi.org/10.1016/j.chaos.2020. 110495 .

[12] P. Afshar, S. Heidarian, F. Naderkhani, A. Oikonomou, K. N. Plataniotis, and A. Mohammadi, "COVID-CAPS: A capsule networkbased framework for identification of COVID-19 cases from X-ray images," Pattern Recognition Letters, vol. 138, pp. 638-643, Oct. 2020, https://doi.org/10.1016/j.patrec.2020.09.010.

[13] A. Saleh, "A Deep Neural Network to Distinguish COVID-19 from other Chest Diseases Using X-ray Images," Current Medical Imaging, vol. 17, no. 1, pp. 109-119, Dec. 2020, https://doi.org/10.2174/15734056166662 00604163954.

[14] P. Sethy, K. Santi, Behera, P. Kumar, and P. Biswas, "Detection of coronavirus Disease (COVID-19) based on Deep Features and Support Vector Machine," vol. 5, no. 4, pp. 643-651, Apr. 2020, https://doi.org/ 10.33889/IJMEMS.2020.5.4.052.

[15] W. Helali, Z. Hajaiej, and A. Cherif, "Real Time Speech Recognition based on PWP Thresholding and MFCC using SVM," Engineering, Technology \& Applied Science Research, vol. 10, no. 5, pp. 6204-6208, Oct. 2020, https://doi.org/10.48084/etasr.3759.

[16] A. Al-Ghraibah, M. Algharibeh, W. Al-Muhtaseb, F. Al-Khateeb, and I. Al-Anis, "Investigating the Significance of New Features Extracted from Long Bones X-ray Images," in 2020 IEEE 5th Middle East and Africa Conference on Biomedical Engineering (MECBME), Amman, Jordan, Oct. 2020, https://doi.org/10.1109/MECBME47393.2020.9265163.

[17] R. C. Gonzalez and R. E. Woods, Digital image processing. New York, NY, USA: Pearson, 2018.

[18] A. A. Alasadi, T. H. Aldhayni, R. R. Deshmukh, A. H. Alahmadi, and A. S. Alshebami, "Efficient Feature Extraction Algorithms to Develop an Arabic Speech Recognition System," Engineering, Technology \& Applied Science Research, vol. 10, no. 2, pp. 5547-5553, Apr. 2020, https://doi.org/10.48084/etasr.3465.

[19] S. Gupta, J. Jaafar, W. F. wan Ahmad, and A. Bansal, "Feature Extraction Using MFCC," Signal \& Image Processing : An International Journal, vol. 4, no. 4, pp. 101-108, Aug. 2013, https://doi.org/10.5121/ sipij.2013.4408.

[20] F. S. Cabral, H. Fukai, and S. Tamura, "Feature Extraction Methods Proposed for Speech Recognition Are Effective on Road Condition Monitoring Using Smartphone Inertial Sensors," Sensors, vol. 19, no. 16, Jan. 2019, Art. no. 3481, https://doi.org/10.3390/s19163481.

[21] T. Grzywalski et al., "Parameterization of Sequence of MFCCs for DNN-based voice disorder detection," in 2018 IEEE International Conference on Big Data (Big Data), Seattle, WA, USA, Dec. 2018, pp. 5247-5251, https://doi.org/10.1109/BigData.2018.8622012.
[22] T. M. Mitchell, Machine learning. New York, NY, USA: Mcgraw Hill, 2017

[23] A. Al-Ghraibah, L. E. Boucheron, and R. T. J. McAteer, "An automated classification approach to ranking photospheric proxies of magnetic energy build-up," Astronomy \& Astrophysics, vol. 579, Jun. 2015, Art. No. A64, https://doi.org/10.1051/0004-6361/201525978.

[24] M Tamer Ozsu and L. Liu, Encyclopedia of database systems. New York, NY, USA: Springer, 2009.

[25] A. Tharwat, "Classification assessment methods," Applied Computing and Informatics, vol. 17, no. 1, pp. 168-192, Jan. 2020, https://doi.org/ 10.1016/j.aci.2018.08.003.

[26] M. Kalechman, Practical MATLAB basics for engineers. Boca Raton, FL, USA: Crc Press, 2018

[27] "Chest X-Ray Images (Pneumonia)," Kaggle. https://kaggle.com/ paultimothymooney/chest-xray-pneumonia (accessed Jun. 03, 2021). 